\title{
An eyelid swelling with a parotid mass
}

\author{
V Seenu, MC Misra, RK Khazanchi, Arun Kumar Goel, MB Prakash
}

All India Institute of Medical Sciences, New Delhi, India 110029 Department of Surgical Disciplines,

V Seenu

MC Misra

RK Khazanchi

AK Goel

Department of

Pathology

MB Prakash

Correspondence to

Dr MC Misra

Accepted 12 December 1995
A 45-year-old woman presented with a left upper eyelid mass, first noticed five years earlier. The mass had been excised initially but recurred within nine months. For the last year, the patient had noticed a painless, progressively increasing swelling in the left parotid region. Examination revealed a $3 \times 2 \mathrm{~cm}$ nontender mass in the left upper eyelid with conjunctival congestion (figure). The mass was subcutaneous in location and adherent to the overlying scar of previous surgery. Visual acuity was normal. There was also a $10 \times 8 \mathrm{~cm}$ firm nontender mass in the left parotid area. Facial nerve function was not impaired.

Liver function tests (serum alkaline phosphatase, alanine transaminase, aspartate transaminase and bilirubin), chest X-ray and ultrasound scan of the liver were normal.

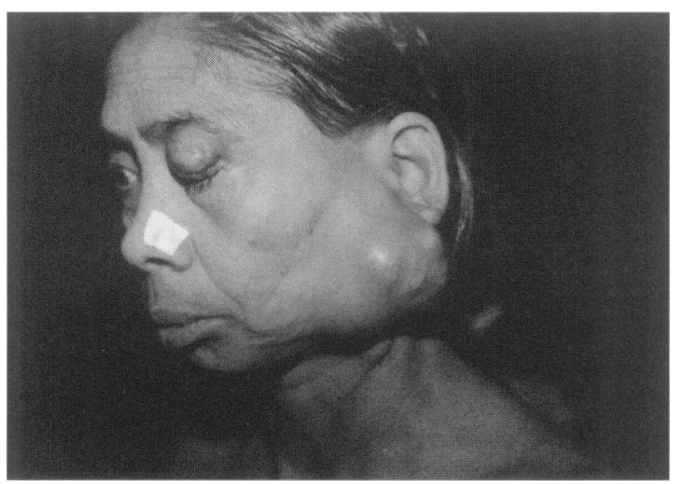

\section{Questions}

1 What is the differential diagnosis?

2 What diagnostic test would you perform?

Figure Clinical photograph of patient showing a small mass in the left upper eyelid and a larger mass in the left parotid region 


\section{Answers}

\section{QUESTION 1}

Based on a history of a recurrent left eyelid mass and a mass in the left parotid area, a malignant tumour of the eyelid, with local recurrence after inadequate primary surgery and metastases to regional lymph node in the parotid area, is the probable diagnosis.

Malignant tumours that need to be considered in this case are cutaneous malignancies of the eyelid such as basal cell carcinoma, squamous cell carcinoma and malignant melanoma, or tumours of the orbital adnexa. Since the tumour was subcutaneous on examination, malignancies of the orbital adnexa become the most likely possibility. Among the tumours of orbital adnexa, meibomian gland carcinoma (sebaceous carcinoma of the eyelid) is the most common.

The possibility of two unrelated pathologies also needs to be considered (a benign eyelid swelling such as chalazion, which can recur after excision and a primary parotid tumour) as primary parotid tumours are much more common. Only $10 \%$ of parotid tumours are secondary. ${ }^{1}$

\section{QUESTION 2}

For a suspected malignancy of the parotid or metastases to the parotid lymph nodes, fine needle aspiration cytology is the procedure of choice. This technique has a very high accuracy in diagnosis of malignancy and may often provide information about the type of tumour. Since the eyelid mass was also large, fine needle aspiration cytology could be carried out from both the masses in this case.

\section{Discussion}

Fine needle aspiration cytology revealed an adenocarcinoma from the eyelid swelling as well as the parotid mass. This observation coupled with the subcutaneous location of the eyelid swelling, excluded primary cutaneous malignancies from the differential diagnosis. Wide excision of the eyelid tumour with eyelid reconstruction and left superficial parotidectomy was performed. Histopathological examination revealed an undifferentiated carcinoma of the eyelid with metastases to the parotid gland. Fat stain results were positive, confirming the diagnosis of sebaceous carcinoma.

Postoperative adjuvant radiotherapy to the parotid area was advised but the patient refused. Eight months later, the tumour recurred in the left parotid region but there was no recurrence in the eyelid. The recurrent tumour was fixed and unsuitable for surgery. Palliative radiotherapy was administered for relief of symptoms.

The parotid gland may be secondarily involved by malignant disease arising at other sites (box 1). Secondary spread to the parotid is usually through the lymphatic route. Many lymph nodes are present in the pre-auricular area, on the surface and within the parotid gland, and drain superficial tissues from the scalp and face. The usual primary tumours are

\section{Parotid secondaries}

- only $10 \%$ of parotid tumours are metastatic

- $90 \%$ of them arise from the head and neck area through lymphatic spread

- the usual primary tumours are cutaneous malignancies, orbital adnexal tumours and mucosal tumours

- $10 \%$ of metastases are haematogenous from distant primary sites such as breast, lung or kidney

Box 1

Meibomian carcinoma (sebaceous carcinoma of the eyelid)

- constitutes $1-5 \%$ of all eyelid malignancies

- commonly occurs in old age and females

- upper eyelid involvement is commoner

- often misdiagnosed as a chalazion

- histologically an adenocarcinoma, staining positively with fat stains such as Oil Red O and Sudan Black

Box 2

squamous cell carcinoma, melanoma and basal cell carcinoma but mucosal malignancies can also involve these nodes. ${ }^{2}$

Sebaceous carcinoma of the eyelids ${ }^{3-5}$ is uncommon (box 2). Metastases to parotid or other cervical lymph nodes may occur. A diagnosis of parotid metastases from sebaceous carcinoma of eyelid may be suspected in a patient who has or has had an eyelid mass.

Management of sebaceous carcinoma metastatic to the parotid gland is not standardised but most authors ${ }^{3-5}$ recommend a superficial parotidectomy as the local procedure of choice because lymph nodes in the parotid gland are almost always confined to the superficial lobe. ${ }^{6}$ In retrospect, however, we feel that total parotidectomy preserving the facial nerve may be a better choice if the metastatic lesions are large. In addition to parotidectomy, dissection of neck nodes has been recommended by some authors. ${ }^{4,5}$ Postoperative radiotherapy to the parotid bed has also been advised with a view to reducing local recurrences. ${ }^{3-5}$ The contribution of neck dissection and postoperative radiotherapy to outcome is not well defined and needs further evaluation. The prognosis of patients with parotid metastases from sebaceous carcinoma is not well documented but up to $50 \%$ of treated patients may survive for five years. Local recurrence is a major problem and some patients also develop distant metastases to lungs, brain, etc.

\section{Final diagnosis}

Sebaceous carcinoma of the eyelid with parotid metastases.

Keywords: meibomian carcinoma, eyelid neoplasm, parotid neoplasm 
1 Grage TB, Lober PH. Malignant tumours of the major salivary glands. Surgery 1962; 52: 284-94.

2 Batsakis JG, Bautina E. Metastases to major salivary glands. Ann Otol Rhinol Laryngol 1990; 99: 501-3.

3 Ginsberg J. Present status of meibomian gland carcinoma. Arch Ophthalmol 1965; 73: $271-7$.

4 Kluka E, David SK Jr, Lyons DG. Meibomian gland Kluka E, David SK Jr, Lyons DG. Meibomian gland
adenocarcinoma of the eyelid metastasising to parotid gland. Ear Nose Throat f 1991; 70: $502-4$
5 Mashburn MA, Chonkich GD, Chase DR, Linda H7=4. Meibomian gland adenocarcinoma of the eyelid with preauricular lymph node metastasis Laryngoscope 1985; 95: $1441-3$.

6 McKean ME, Lee K, McGregor IA. The distribution of lymph nodes in and around the parotid gland: an anatomical study. Br f Plast Surg 1985; 38: 1-5.

\title{
Splenomegaly
}

\author{
H Al-Omishy, C Lockett, JA Rees, BR Davidson
}

\section{Department of} Surgery, Royal Free Hospital and Medical School, Pond Street, London NW3 2QG, UK $\mathrm{H}$ Al-Omishy

C Lockett

BR Davidson

Department of

Histopathology

JA Rees

Correspondenceto $\mathrm{Mr} \mathrm{BR}$ Davidson

Accepted 12 December 1995
A 64-year-old man presented with left-sided abdominal pain. He also complained of pain in his right shoulder and pelvis. A liposarcoma had been excised from his left sole six years earlier followed by local radiotherapy. There was no history of exposure to carcinogens. On clinical examination he had splenomegaly but no other significant findings. His blood profile showed a normochromic normocytic anaemia with normal white cells and platelets. Liver function tests and coagulation profile were normal. Ultrasound examination confirmed the splenomegaly. A computed tomography (CT) scan of the abdomen is shown in the figure. A bone scan was suggestive of metastatic disease.

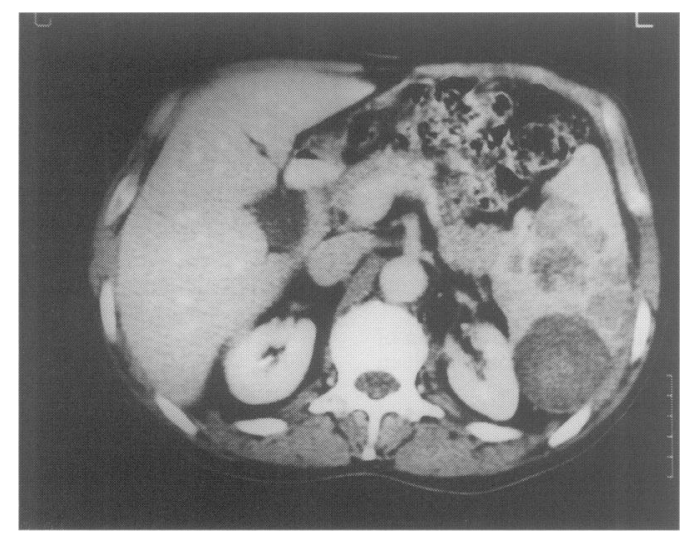

\section{Questions}

1 What is the abnormality shown?

2 How may the diagnosis be established?

Figure CT scan of the abdomen 